\title{
Balancing with balancing powers
}

\author{
Nurettin Irmak
}




\title{
BALANCING WITH BALANCING POWERS
}

\author{
NURETTIN IRMAK
}

Received 21 March, 2013

Abstract. In this paper, the Diophantine equation $B_{1}^{k}+B_{2}^{k}+\cdots+B_{n-1}^{k}=B_{n+1}^{l}+B_{n+2}^{l}+$ $\cdots+B_{n+r}^{l}$ for the positive integer unknowns $n \geq 2, k, l$ and $r$ is studied in certain cases, where $B_{n}$ denotes the $n^{t h}$ term of the balancing sequence.

2010 Mathematics Subject Classification: 11B39

Keywords: balancing numbers, powers

\section{INTRODUCTION}

The first definition of balancing numbers is essentially due to Finkelstein [5], though he named them numerical centers. A positive integer $n \geq 2$ is called balancing number if

$$
1+2+\cdots+(n-1)=(n+1)+(n+2)+\cdots+(n+r)
$$

holds for some positive integer $r$ which is called balancer corresponding to the balancing number $n$. The $m^{t h}$ term of the sequence of balancing numbers is denoted by $B_{m}$. It is known that the balancing numbers satisfy the recurrence

$$
B_{n}=6 B_{n-1}-B_{n-2}
$$

where initial contidions are defined by $B_{0}=0$ and $B_{1}=1$. The first few terms of the balancing sequence are

$$
0,1,6,35,204,1189,6930,40391, \ldots \text {. }
$$

The Binet formula of the balancing numbers is given by

$$
B_{n}=\frac{\alpha^{n}-\beta^{n}}{\alpha-\beta}=\frac{(3+2 \sqrt{2})^{n}-(3-2 \sqrt{2})^{n}}{4 \sqrt{2}},
$$

where $\alpha$ and $\beta$ are the roots of the chaeacteristic polynomial $x^{2}-6 x+1$ of the recursive sequence (1.2).

Behera et al. [4] showed that the diophantine equation

$$
F_{1}^{k}+F_{2}^{k}+\cdots+F_{n-1}^{k}=F_{n+1}^{l}+F_{n+2}^{l}+\cdots+F_{n+r}^{l}
$$


has no solution in the positive integers $n, r, k, l$ with $n \geq 2$ in the case

$$
k \leq l \text { and }(k, l)=(2,1),(3,1),(3,2) .
$$

Here $F_{n}$ denotes the $n^{\text {th }}$ Fibonacci number. They also conjectured in [4] that only the quadruple $(n, r, k, l)=(4,3,8,2)$ satisfies (1.3). Their conjecture was proved by Alvarado et al. [2].

In this paper, we focus on the diophantine equation

$$
B_{1}^{k}+B_{2}^{k}+\cdots+B_{n-1}^{k}=B_{n+1}^{l}+B_{n+2}^{l}+\cdots+B_{n+r}^{l} .
$$

In the specific cases (1.4) we found no solutions. Based on Theorems 6-9 and an extended computer search, we conjectured the following.

Conjecture 1. There is no quadruple $(n, r, k, l)$ of positive integers $(n \geq 2)$ which satisfies (1.5).

\section{LEMMAS}

The proofs of the theorems use several statements collected in this section.

Lemma 1. For any positive integer $m$

(a): $B_{m+1} B_{m-1}=\left(B_{m}+1\right)\left(B_{m}-1\right)$,

(b): $B_{2 m-1}=B_{m}^{2}-B_{m-1}^{2}$,

(c): $B_{1}+B_{3}+\cdots+B_{2 m-1}=B_{m}^{2}$,

(d): $B_{1}^{2}+B_{2}^{2}+\cdots+B_{m-1}^{2}<B_{m+1}+B_{m+2}+\cdots+B_{2 m-3}$ for $m \geq 5$,

(e): $B_{2 m+5}^{2}+B_{2 m+6}^{2}+\cdots+B_{3 m+4}^{2}<B_{1}^{3}+B_{2}^{3}+\cdots+B_{2 m+3}^{3}$ for $m \geq 2$,

(f): $4 B_{m}<B_{m+1}-B_{m}<5 B_{m}$

hold.

Proof. The first three identities appear in [3] and [6].

The fourth property (d) can be justified by induction on $m$. Clearly, $B_{1}^{2}+B_{2}^{2}+$ $B_{3}^{2}+B_{4}^{2}<B_{6}+B_{7}$. Now suppose that,

$$
B_{1}^{2}+B_{2}^{2}+\cdots+B_{m-1}^{2}<B_{m+1}+B_{m+2}+\cdots+B_{2 m-3} .
$$

Evaluating

$$
B_{1}^{2}+B_{2}^{2}+\cdots+B_{m-1}^{2}+B_{m}^{2}<B_{m+1}+B_{m+2}+\cdots+B_{2 m-3}+B_{m}^{2}
$$

we see that $B_{m}^{2}<B_{2 m-1}+B_{2 m-2}-B_{m+1}$ fulfills, and then

$$
\begin{aligned}
B_{1}^{2}+B_{2}^{2}+\cdots+B_{m}^{2} & <B_{m+1}+B_{m+2}+\cdots+B_{2 m-3}+B_{2 m-1}+B_{2 m-2}-B_{m+1} \\
& =B_{m+2}+B_{m+3}+\cdots+B_{2 m-1}
\end{aligned}
$$

follow. Indeed, $B_{m}^{2}-B_{2 m-1}=B_{m-1}^{2}<B_{2 m-2}-B_{m-1}$.

The identity (e) can be seen by the same way, we leave it to the readers, as well as the proof of the statement (f).

Lemma 2. Any positive integer $n$ satisfies 
(a): $\sum_{k=1}^{n} B_{k}=\left(B_{n+1}-B_{n}-1\right) / 4$,

(b): $\sum_{k=1}^{n} B_{k}^{2}=\left(B_{2 n+1}-(2 n+1)\right) / 32$,

(c): $\sum_{k=1}^{n} B_{k}^{3}=\left(B_{3 n+3}-B_{3 n}-147\left(B_{n+1}-B_{n}\right)+112\right) / 6272$.

Proof. Since the technique of the proofs of the cases (a), (b) and (c) coincide, we omit the first statement and start with the second one.

(b) By the Binet formula of the balancing numbers,

$$
\begin{aligned}
\sum_{k=1}^{n} B_{k}^{2} & =\frac{1}{(\alpha-\beta)^{2}} \sum_{k=0}^{n-1} \alpha^{2 k+2}+\beta^{2 k+2}-2 \\
& =\frac{1}{32}\left(\alpha^{2} \frac{1-\alpha^{2 n}}{1-\alpha^{2}}+\beta^{2} \frac{1-\beta^{2 n}}{1-\beta^{2}}-2 n\right) \\
& =\frac{1}{32}\left(\frac{\alpha^{2 n+2}+\beta^{2 n+2}-\left(\alpha^{2 n}+\beta^{2 n}\right)}{32}-(2 n+1)\right) \\
& =\left(B_{n+1}^{2}-B_{n}^{2}-(2 n+1)\right) / 32 \\
& =\left(B_{2 n+1}-(2 n+1)\right) / 32 .
\end{aligned}
$$

(c) For the proof of the third identity, we use the Binet formula of balancing numbers again as follows.

$$
\begin{aligned}
\sum_{k=1}^{n} B_{k}^{3} & =\frac{1}{(\alpha-\beta)^{3}} \sum_{k=0}^{n-1} \alpha^{3(k+1)}-\beta^{3(k+1)}-3\left(\alpha^{k+1}-\beta^{k+1}\right) \\
& =\frac{1}{(\alpha-\beta)^{3}}\left\{\alpha^{3} \frac{1-\alpha^{3 n}}{1-\alpha^{3}}-\beta^{3} \frac{1-\beta^{3 n}}{1-\beta^{3}}-3\left(\alpha \frac{1-\alpha^{n}}{1-\alpha}-\beta \frac{1-\beta^{n}}{1-\beta}\right)\right\} \\
& =\frac{1}{32}\left\{\frac{B_{3(n+1)}-B_{3 n}-35}{196}-3 \frac{B_{n+1}-B_{n}-1}{4}\right\} \\
& =\frac{B_{3 n+3}-B_{3 n}-147\left(B_{n+1}-B_{n}\right)+112}{6272} .
\end{aligned}
$$

and the proof is completed.

Lemma 3. For any integer $u \geq 3$, the inequalities

$$
\alpha^{u-0.99}<B_{u}<\alpha^{u-0.98}
$$

hold.

Proof. See Lemma 4 in [1].

Lemma 4. Suppose that $a>0$ and $b \geq 0$ are real numbers and $u_{0}$ is a positive real number. Then $a \alpha^{u}+b \leq \alpha^{u+\kappa}$ holds for any $u \geq u_{0}$ where $\kappa=\log _{\alpha}\left(a+\frac{b}{\alpha^{u_{0}}}\right)$.

Proof. For the proof, see Lemma 2.4 in [4]. 


\section{THE RESULTS}

Here we present four theorems and their proofs which confirm Conjecture 1.

Theorem 1. The diophantine equation $B_{1}^{k}+B_{2}^{k}+\cdots+B_{n-1}^{k}=B_{n+1}^{l}+B_{n+2}^{l}+$ $\cdots+B_{n+r}^{l}$ has no solution for any positive integers $r$ and $n \geq 2$ if $k \leq l$.

Proof. Applying Lemma 2(a) for $k \leq l$, we get

$$
\begin{aligned}
B_{1}^{k}+B_{2}^{k}+\cdots+B_{n-1}^{k} & \leq\left(B_{1}+B_{2}+\cdots+B_{n-1}\right)^{k}=\left(\frac{B_{n}-B_{n-1}-1}{4}\right)^{k} \\
& \leq\left(\frac{B_{n}-B_{n-1}-1}{4}\right)^{l}<B_{n}^{l},
\end{aligned}
$$

which completes the proof of Theorem 1.

Theorem 2. The diophantine equation $B_{1}^{2}+B_{2}^{2}+\cdots+B_{n-1}^{2}=B_{n+1}+B_{n+2}+$ $\cdots+B_{n+r}$ has no solution in positive integers $r$ and $n \geq 2$.

Proof. By the virtue of Lemma 2(a) and (b), the equation

$$
B_{1}^{2}+B_{2}^{2}+\cdots+B_{n-1}^{2}=B_{n+1}+B_{n+2}+\cdots+B_{n+r}
$$

is equvalent to

$$
B_{2 n-1}+8\left(B_{n+1}-B_{n}\right)-(2 n-1)=8\left(B_{n+r+1}-B_{n+r}\right) .
$$

For brevity, we use the following labels:

$$
\begin{aligned}
& L S:=B_{2 n-1}+8\left(B_{n+1}-B_{n}\right)-(2 n-1) \\
& R S:=8\left(B_{n+r+1}-B_{n+r}\right) .
\end{aligned}
$$

Using Lemma 1(f),

$$
B_{2 n-1}<L S<B_{2 n-1}+40 B_{n}
$$

hold. The by Lemma 3,

$$
\alpha^{2 n-1.99}<L S<\alpha^{2 n-1.98}+40 \alpha^{n-0.98} \leq \alpha^{2 n-1.98}+\alpha^{n+1.12} .
$$

According to the Lemma 4, if $a=b=1$ we obtain $\kappa<0.02$. Thus

$$
\alpha^{2 n-1.99}<L S<\alpha^{n+1.12}\left(\alpha^{n-3.1}+1\right)<\alpha^{n+1.12} \alpha^{n-3.1} \alpha^{0.02} .
$$

Consequently,

$$
\alpha^{2 n-1.99}<L S<\alpha^{2 n-1.96} .
$$

On the other hand, Lemma 1(f) implies

$$
32 B_{n+r}<R S<40 B_{n+r}
$$

and then

$$
\alpha^{n+r-0.99} \alpha^{1.95}=\alpha^{n+r+0.96}<R S<\alpha^{n+r+1.12}=\alpha^{n+r-0.98} \alpha^{2.1}
$$


follow. Thus, from (3.2) and (3.3) we get

$$
\max \{2 n-1.99, n+r+0.96\}<\min \{2 n-1.96, n+r+1.12\}
$$

which yields the inequalities

$$
2 n-1.99<n+r+1.12
$$

and

$$
n+r+0.96<2 n-1.96 .
$$

Hence, the positive integer $n$ and $r$ satisfy

$$
n-3.11<r<n-2.92 \text {, }
$$

that is $r=n-3$. If we plug $r=n-3$ in (3.1), we obtain

$$
B_{1}^{2}+B_{2}^{2}+\cdots+B_{n-1}^{2}=B_{n+1}+B_{n+2}+\cdots+B_{2 n-3}
$$

which contradicts to Lemma $1(\mathrm{~d})$.

Theorem 3. The diophantine equation $B_{1}^{3}+B_{2}^{3}+\cdots+B_{n-1}^{3}=B_{n+1}+B_{n+2}+$ $\cdots+B_{n+r}$ has no solution for any positive integers $r$ and $n \geq 2$.

Proof. Obviously, $n=2$ the case provides no solution. Therefore we may assume $n \geq 3$, By Lemma 2(a) and (c), the equation in the theorem is equivalent to

$B_{3 n}-B_{3(n-1)}+1568 B_{n+1}-1715 B_{n}+147 B_{n-1}+112=1568\left(B_{n+r+1}-B_{n+r}\right)$.

Using the labels again, put

$$
\begin{aligned}
& L S:=B_{3 n}-B_{3(n-1)}+1568 B_{n+1}-1715 B_{n}+147 B_{n-1}+112, \\
& R S:=1568\left(B_{n+r+1}-B_{n+r}\right) .
\end{aligned}
$$

By Lemma 3, it is obvious that

$$
196 B_{3(n-1)}<L S<197 B_{3(n-1)} .
$$

Further we have

$$
\alpha^{3 n-3.99} \alpha^{2.98}=\alpha^{3 n-1.01}<L S<\alpha^{3 n-0.98}=\alpha^{3 n-3.98} \alpha^{3.00}
$$

On the other hand, applying Lemma 1(f),

$$
\alpha^{n+r+3.96}=1568 \cdot 4 \cdot B_{n+r}<R S<1568 \cdot 5 B_{n+r}=\alpha^{n+r+4.11}
$$

hold. From (3.6) and (3.7) we get

$$
\max \{3 n-1.01, n+r+3.96\}<\min \{3 n-0.98, n+r+4.11\},
$$

and we deduce

$$
2 n-5.12<r<2 n-4.94 .
$$

Since $n$ and $r$ are integers in this inequality, only $r=2 n-5$ is possible (recall that $n \geq 3$ ). But it contradicts to

$$
B_{3 n}-B_{3(n-1)}+1568 B_{n+1}-1715 B_{n}+147 B_{n-1}+112<1568\left(B_{3 n-4}-B_{3 n-5}\right) \text {. }
$$


Therefore, there is no solution which satisfies (3.5).

Theorem 4. The diophantine equation $B_{1}^{3}+B_{2}^{3}+\cdots+B_{n-1}^{3}=B_{n+1}^{2}+B_{n+2}^{2}+$ $\cdots+B_{n+r}^{2}$ has no solution for any positive integers $r$ and $n \geq 2$.

Proof. For $2 \leq n \leq 4$, th statement is obvious. Assume now $n \geq 5$. The application of Lemma 2(b)and (c) converts the equation

$$
B_{1}^{3}+B_{2}^{3}+\cdots+B_{n-1}^{3}=B_{n+1}^{2}+B_{n+2}^{2}+\cdots+B_{n+r}^{2}
$$

to

$$
\frac{B_{3 n}-B_{3(n-1)}-147\left(B_{n}-B_{n-1}\right)+112}{32 \cdot 196}=\frac{1}{32}\left(B_{2(n+r)+1}-B_{2 n+1}-2 r\right),
$$

which is equivalent to

$$
B_{3 n}-B_{3(n-1)}-147\left(B_{n}-B_{n-1}\right)+196 B_{2 n+1}+112=196\left(B_{2(n+r)+1}-2 r\right) .
$$

Put

$$
\begin{aligned}
& L S:=B_{3 n}-B_{3(n-1)}-147\left(B_{n}-B_{n-1}\right)+196 B_{2 n+1}+112, \\
& R S:=196\left(B_{2(n+r)+1}-2 r\right) .
\end{aligned}
$$

Now use Lemma 3 and Lemma 1(f) to obtain

$$
\alpha^{2 n+2 r+3}<R S<\alpha^{2 n+2 r+3.02} .
$$

Similarly,

$$
\begin{aligned}
196 B_{3(n-1)} & <L S<(197+196) B_{3(n-1)}, \\
\alpha^{3 n-3-0.99} \alpha^{2.98} & =\alpha^{3 n-1.01}<L S<\alpha^{3 n-0.59}=\alpha^{3 n-3-0.98} \alpha^{3.39}
\end{aligned}
$$

hold. From (3.9) and (3.10)

$$
\max \{2 n+2 r+3,3 n-1.01\}<\min \{2 n+2 r+3.02,3 n-0.59\}
$$

follows, which yields

$$
n-4.03<2 r<n-3.59 .
$$

Clearly, only $2 r=n-4$ is possible. Inserting $n=2 r+4$ to (3.8), we obtain

$$
B_{1}^{3}+B_{2}^{3}+\cdots+B_{2 r+3}^{3}=B_{2 r+5}^{2}+B_{2 r+6}^{2}+\cdots+B_{3 r+4}^{2},
$$

and we arrived at a contradiction with Lemma 1(e).

\section{ACKNOWLEDGEMENT}

The authors would like to thank László Szalay for his helpful suggestions. 


\section{REFERENCES}

[1] M. Alp, N. Irmak, and L. Szalay, "Balancing diophantine triples," Acta Univ. Sapientiae, Math., vol. 4, no. 1, pp. 11-19, 2012.

[2] S. D. Alvarado, A. Dujella, and F. Luca, "On a conjecture regarding balancing with powers of Fibonacci numbers," Integers, vol. 12, no. 6, pp. 1127-1158, a2, 2012.

[3] A. Behera and G. K. Panda, "On the square roots of triangular numbers," Fibonacci Q., vol. 37, no. 2, pp. $98-105,1999$.

[4] A. Behera, K. Liptai, G. K. Panda, and L. Szalay, "Balancing with Fibonacci powers," Fibonacci Q., vol. 49, no. 1, pp. 28-33, 2011.

[5] R. Finkelstein, "The house problem," Am. Math. Mon., vol. 72, pp. 1082-1088, 1965.

[6] G. K. Panda, "Some fascinating properties of balancing numbers," Congr. Numerantium, vol. 194, pp. 185-189, 2009.

Author's address

Nurettin Irmak

Niğde University, Art and Science Faculty, Mathematics Department, 51110 Niğde, TURKEY

E-mail address: nirmak@nigde.edu.tr 